\title{
Queue de renard, ficelle et plume : classer sa bibliothèque
}

Le cas de Pierre de L'Estoile : 1606 à 1611

\section{Gilbert Schrenck}

\section{OpenEdition}

\section{Journals}

Édition électronique

URL : http://journals.openedition.org/aes/1569

DOI : $10.4000 /$ aes. 1569

ISSN : 2258-093X

Éditeur

Laboratoire LISAA

\section{Référence électronique}

Gilbert Schrenck, "Queue de renard, ficelle et plume : classer sa bibliothèque », Arts et Savoirs [En

ligne], 10 | 2018, mis en ligne le 21 novembre 2018, consulté le 02 mai 2019. URL : http://

journals.openedition.org/aes/1569; DOI : 10.4000/aes.1569

Ce document a été généré automatiquement le 2 mai 2019.

Centre de recherche LISAA (Littératures SAvoirs et Arts) 


\title{
Queue de renard, ficelle et plume : classer sa bibliothèque
}

\author{
Le cas de Pierre de L'Estoile : 1606 à 1611
}

\section{Gilbert Schrenck}

1 Selon l'inventaire après décès établi par Jacques Douceur entre le 25 octobre et le 21 novembre 1611, la bibliothèque de Pierre de L'Estoile comprendrait un ensemble de 2632 ouvrages, tous genres confondus ${ }^{1}$. Que ce chiffre ne soit qu'approximatif ne change rien à l'importance du fonds qui classe la «librairie " parmi les plus importantes de son siècle 2 . Elle contient, en effet, un nombre impressionnant d'ouvrages, de manuscrits et de «ramas » qui font se côtoyer, parmi d'autres curiosités, des œuvres prestigieuses et des pièces de moindre valeur, telles les «bagatelles » et «fadaises », dont le collectionneur se montre particulièrement friand. Cette étroite proximité de textes appartenant à des sphères culturelles très contrastées, allant des traités les plus érudits aux pièces populaires, annonce d'une certaine manière l'art de « dresser une bibliothèque » qui sera bientôt théorisé par Gabriel Naudé et qui recommande justement de n'exclure aucun type d'ouvrages de l'entreprise ${ }^{3}$. C'est justement sur cet aspect d'une classification générale que nous voudrions insister en analysant la manière dont L'Estoile reconfigure son dépôt à l'extrême fin de sa vie et en consigne les étapes dans son Journal ${ }^{4}$. C'est effectivement l'époque où, en bibliophile aguerri, il trie, cote et classe ses livres à travers une série de gestes et d'observations qui instruisent avec précision sur les aspects de la bibliographique matérielle en une série de considérations assez rares chez un amateur des lettres $d u \mathrm{XVI}^{\mathrm{e}}$ siècle pour ne pas être signalées. Ses remarques, dont on pressent qu'elles relèvent aussi de l'urgence d'un homme talonné par la mort ${ }^{5}$, saturent le Journal et fournissent un témoignage précieux sur les activités quasi quotidiennes et fortement ritualisées, dont le mémorialiste entoure l'objet de sa passion. Inutile de dire que son image de bibliothécaire au quotidien se situe loin de la pose figée et quelque peu hiératique que l'iconographie humaniste - on pense au portrait d'Érasme peint par Holbein -, représente. Avec L'Estoile, les postures d'un individu qui classe sa bibliothèque avec application sont d'allure ordinaire. 
2 Face à l'ampleur des notes relatives à ce sujet, il est nécessaire d'en limiter le nombre à la période des années 1606 à 1611, durant laquelle L'Estoile s'affaire de manière compulsive à la réalisation de son projet. À cette époque, le chaos des guerres civiles appartient au passé, même si l'assassinat d'Henri IV, le 14 mai 1610, par Ravaillac, pouvait un moment laisser craindre le pire. Globalement, le temps est venu d'ordonner sa collection de médailles, de livres et d'autres objets qui garnissent son cabinet de curiosités, profitant du loisir que lui laissait la vente de sa charge de Grand audiencier à la chancellerie de Paris pour lire Montaigne, son vade mecum. Celui-ci, dont les préceptes émaillent le Journal à partir de l'année 1606, aiguise, en effet, le regard de sagesse que le diariste jette dorénavant sur ses «registres (que j'appelle le magazin de mes curiosités) ». Il l'encourage dans l'opinion désabusée qu'il se fait du spectacle de la vanité humaine, justifie son désir de liberté que procure l'écriture, tout en érigeant un rempart face aux affronts de l'existence ${ }^{7}$. La «librairie» devient progressivement un refuge pour le vieillard ruiné, à la santé défaillante :

Cette année 1609, critique de mon aage, soixante-trois ans, a esté en beaucoup de sorte malencontreuse pour moy et pour les miens, affligé en icelle de diverses maladies de corps et d'esprit, fortuné en mes biens de pertes nouvelles et extraordinaires, travaillé d'affaires et de procès, rejetté de mes proches, mesprisé et inquiété de tous, jusques à des faquins, valets et chambrières. Et qu'y a-t-il, je vous prie, au monde, de plus misérable qu'une vieillesse infirme et nécessiteuse ? $^{8}$

\section{Fournitures}

3 À lire le Journal, on est tout d'abord frappé par les nombreuses mentions faites aux conditions dans lesquelles le mémorialiste s'occupe de ses livres. Le quotidien, dans ce qu'il a de plus banal, se présente aux yeux du lecteur et lui livre, à l'appui d'un grand luxe de détails, l'image du collectionneur affairé par son dispositif de rangement. Par sa profusion, la description très réaliste des gestes introduit au cœur battant du cabinet d'étude où le bibliophile passionné aménage son espace. Sous son impulsion, la bibliothèque prend vie et s'anime grâce à l'empressement du propriétaire attentif à l'achat de fournitures, veillant aux visites chez les libraires et imprimeurs, soucieux aussi de recevoir les curieux et les érudits qui se pressent à sa porte, scrupuleux dans le recrutement de secrétaires et de copistes chargés du collationnement des manuscrits et $\mathrm{du}$ traitement des libelles. Indications toutes indispensables, si l'on cherche à reconstituer la matérialité des lieux et des actions ${ }^{9}$ et qui, plus modestement, informent sur les conditions d'achat d'accessoires soigneusement noté par souci comptable : papier, plumes, encre, ficelles, poudre et parfois une « queue de renard à nettoyer » les livres ${ }^{10}$ :

Ce jour, en papier, plumes, ancre et ficelles, ung quart d'escu (14 août 1607, t. VIII, p. 329).

Ce jour, en plumes, ancre, papier et carnet : douze sols (27 août 1607, t. VIII, p. 333).

Ce jour, en papier, plumes et ancres, dix sols huit deniers (28 août 1608, t. IX, p. 120).

Ledit jour, en plumes, poudre, papier et ancre, ung teston (quinze sols six deniers)

(13 novembre 1608, t. IX, p. 162).

4 Ces notes apparemment triviales, mais nécessaires à la bonne gestion au jour le jour de la « libraire ", coexistent avec des remarques qui disent à quel point L'Estoile reste sensible à l'aspect matériel des objets qu'il manipule :

J'ay donné, ce jour, à M. Du Pui, un petit livre de papier de la Chine, couvert d'un

fort beau papier marbré, que je gardois dès longtemps dans mon cabinet. J'en ay 
encore un pareil, tout semblable (15 décembre 1608, t. IX, p. 179) ${ }^{11}$.

Le mecredi $13^{\mathrm{e}}$, j'ay donné à M. D. P. six feuilles de mon papier marbré, beau par excellence, que je lui avois promis, et dont je sçay qu'il est curieux, aussi bien comme moy qui en ay tousjours de réserve en mon cabinet (13 mai 1609, t. IX, p. 264).

\section{Libraires imprimeurs}

Parallèlement à ces occupations, L'Estoile consigne avec le même sens du détail les visites rendues aux libraires imprimeurs de son quartier dans l'idée de s'informer des dernières nouveautés. Une cartographie des lieux permettrait, en l'occurrence, de suivre à la trace le parlementaire durant ses déambulations de boutique en boutique, où, les échanges et les discussions se déroulent dans un climat d'étroite confiance, voire d'amicale complicité. Cette immersion dans le monde de la librairie lui est, à vrai dire, grandement facilitée par son ancien office de Grand audiencier à la chancellerie, grâce auquel il accordait les privilèges d'imprimer. N'avait-il pas, par le passé, signé ès qualité des privilèges pour Abel Langelier, l'éditeur des Essais de 1595, qui deviendra son livre de chevet $^{12}$, comme il le fit pour Jean Houzé en 1599, Claude Berjon ${ }^{13}$ et Gilles Le Beys, l'imprimeur Au Lis Blanc de la rue Saint-Jacques, qui avait fait paraître en 1586 le Souverain remède contre témérité et avarice du carme Pierre Courtin ${ }^{14}$ ?

Les boutiques du Palais de justice et celles du quartier Saint-Jacques sont les endroits habituels des rencontres avec les professionnels ayant pignon sur rue. Là, en ces lieux d'échanges, L'Estoile a l'opportunité de croiser tous ceux qui comptent, ou ont compté un jour, dans la profession, anciens imprimeurs ligueurs, catholiques zélés et protestants actifs, comme Éloi Gibier, Jean Jannon et Thomas Périer. Dans ces espaces, l'amour du livre transcende les clivages confessionnels et les prises de positions partisanes, ainsi que le montre l'exemple des entretiens que le visiteur mène avec ses interlocuteurs sur l'opportunité de publier tel ou tel ouvrage. À Claude Berjon, venu spécialement le consulter à son domicile de la rue des Grands-Augustins sur le projet de mettre sous presse "un petit discours de l'impossibilité des deux religions ", L'Estoile déconseille vivement l'initiative ${ }^{15}$ et reçoit en reconnaissance une demi-douzaine d'exemplaires "d'un petit traité, bien fait, en faveur des États, pour ne quitter le commerce des Indes », dont il n'acceptera finalement que trois spécimens ${ }^{16}$. Un peu plus tard, Berjon lui remet dans le même esprit des copies de libelles ${ }^{17}$. Quant à Jean Berjon, un parent du précédent, "que je congnois, et auquel je désirerois faire plaisir $»^{18}$, le diariste déplore l'incarcération au Châtelet à l'issue de la publication imprudente du libelle satirique, $L e$ Secret des jesuistes. D'autres libraires font également partie de ses généreux amis, comme Toussaint Du Bray qui venait de publier le Discours nouveau des marques de l'Eglise de l'archevêque de Bourges ${ }^{19}$, et Jean Houzé un Avis sur les duels ${ }^{20}$, deux exemples, parmi d'autres, qui disent l'importance des rapports de l'intéressé avec les libraires et qui se concrétisent par des prêts, des dons de livres et des conseils ${ }^{21}$. À lire ces témoignages entre les lignes, ces rencontres sont également révélatrices des graves tensions qui traversent le champ éditorial du début $d u \mathrm{XVII}^{\mathrm{e}}$ siècle et pointent à travers elles la position gallicane de L'Estoile en matière de lectures politiques et de publications sur la réunion des religions. Mais d'une manière plus générale, elles définissent chez le mémorialiste les grands principes fondés sur le partage du savoir humaniste avec le plus grand nombre, puisque L'Estoile n'hésite pas à remettre à Daniel Guillemot une copie de sa Chronique de Saint-Loys en vue d'une réédition ${ }^{22}$ : libéralité d'autant plus remarquable, 
quand elle est reconduite en faveur d'un ancien imprimeur de la Ligue, dont L'Estoile eut pourtant à souffrir : Gilles Robinot, à qui il prête un de ses ouvrages pour être réimprimée ${ }^{3}$

$7 \quad$ Mais au-delà de la fréquentation des boutiques, le quotidien de L'Estoile se meuble aussi de scènes anecdotiques, souvent pittoresques, ou dramatiques, de la vie secrète du cercle des libraires. Un jour, il se décrit en train de «fureter des livres » entre les étals de Jean Ruel, à moins que ce ne soit ceux de René, son frère, quand il tombe par hasard sur « un vieil bouquin ou roman théologique en vers, intitulé : Le Mystere et miracle de monseigneur saint Jacques en la ville de Compostelle en Galice", invraisemblable imbroglio d'amours ancillaires et de miracles bouffons ${ }^{24}$. Une autre fois, dans l'échoppe d'Adrien Périer, il entre en débat avec un gentilhomme portugais à propos des écrits du jésuite Mariana ${ }^{25}$. Toujours dans la même boutique qu'il semble avoir assidument fréquentée, il s'amuse au spectacle quasi carnavalesque d'une bande de capucins espagnols qui passe ridiculement devant la vitrine du libraire ${ }^{26}$. Et c'est chez lui encore qu'il croise le commissaire Langlois venu « faire défense de débiter ni vendre à l'avenir, à quelque personne que ce fût, ni de quelque qualité qu'elle peust estre, le livre d'un jésuiste nommé Sanchez, De Matrimonio, imprimé in-folio, ni mesmes d'en tenir ou avoir en sa boutique ${ }^{27}$. Comble d'ironie, L'Estoile n'hésite pas à recopier sur le champ un extrait de l'ordonnance qui frappait ce traité qu'il tenait, du reste, de longue date dans sa bibliothèque ${ }^{28}$. C'est dire aussi à quel point la question de la censure interpelle le collectionneur au point de le scandaliser, lorsque les interdits émanent des milieux protestants et de leurs ministres, comme ce fut le cas lors d'une visite chez

l'imprimeur Janon, que je cognois il y a longtemps, [et qui] m'a donné, de son impression, la Response faite par Pelletier à l'Apologie du Roy d'Angleterre, avec la conversion dudit sieur [...]. Et pource que Janon, l'imprimeur, a tousjours esté et est encores de la Religion, Messieurs ses maistres de Charanton, aians trouvé fort mauvais de ce qu'il s'estoit ingéré d'imprimer ces livres, directement contraires à la profession et doctrine de leurs Eglises, [...] lui ont interdit à temps l'usage de la Cène, avec deffenses de ne plus vendre de livres à Charanton, comme il y avait accoustumé. $^{29}$

S'il lui arrive de juger, et parfois sévèrement, les gens de la profession ${ }^{30}$, il ne renonce jamais à consulter les catalogues qu'ils rapportent de la Foire de Francfort et qu'il recopie à l'occasion sur ses tablettes ${ }^{31}$. Ses libraires habituels et plus particulièrement Gilles Le Beys, un proche de l'Anversois Plantin, lui procure un recueil de satires latines signalé par le catalogue allemand ${ }^{32}$, geste suivi par Adrien Le Beys qui en fait de même avec l' Epithalame de Hensius fraîchement imprimé à Leyde, puis avec le Mercurius Gallobelgicus ${ }^{33}$.

\section{Copistes}

9 Il faut cependant noter que la restructuration de la bibliothèque monopolise des moyens et des interventions qui ne sauraient se limiter à la seule acquisition de fournitures et de catalogues, ou à des flâneries dans les échoppes parisiennes. La passion livresque est aussi assouvie grâce au concours d'hommes de confiance, secrétaires, copistes et relieurs, qui, par intermittences, secondent le parlementaire dans la gestion et l'ordonnancement du fonds. $\mathrm{M}^{\mathrm{e}} \mathrm{Abraham}$, le relieur attitré de L'Estoile ${ }^{34}$, déploie à ses côtés une intense activité préparatoire de dossiers et de registres, maniant à tour de bras papier, plumes et encre ${ }^{35}$, raccoutrant livres et libelles ${ }^{36}$, inventoriant $"$ paquets et livres curieux $»^{37}$, sans oublier d'informer son maître des derniers ragots de Paris ${ }^{38}$. D'autre part, L'Estoile rémunère « un 
pauvre garson, nommé La Pourie, [de] deux testons, pour m'avoir transcript tout plain de poésies ; aiant en ce plus d'esgard à sa pauvreté qu'à chose que j'en eusse affaire ${ }^{39}$. Si l'amour des livres ne distrait pas de l'amour du prochain, le collectionneur reste tout aussi sensible à la propreté de son étude et de son cabinet de lecture « qui en avoient bon besoin $»^{40}$, soins commis aux bons offices d'un certain Nicolas préposé à cette tâche ${ }^{41}$. Même le maître couvreur Gallot a droit à une mention du Journal « pour avoir recouvert quelques endroits de ma gallerie et cabinet, où il pleuvoit, et qui mesme m'avoit gasté quelques besongnes et livres $\aleph^{42}$. Tout ce petit monde s'active donc dans la bibliothèque, tandis que des copistes mettent au propre et archivent les documents. Guischard transcrit, à l'intention de Dupuy ${ }^{43}$, un des grands amis de L'Estoile, le manuscrit portant la condamnation de Montecuccoli accusé d'avoir empoisonné le dauphin en $1536^{44}$. Et Chausson, ou Chosson, recopie la Harangue que le procureur général La Guesle a prononcée sur l'assassinat d'Henri III ${ }^{45}$. Ce copiste mérite certainement une mention spéciale pour l'extrême générosité que lui témoigne son maître :

Ce jour, aiant eu nouvelles de la mort de son père, [Chosson] reprist le chemin de sa ville de Genève, me remectant fidèlement entre les mains tous les papiers et escritures qu'il avoit à moy et me laissant à achever mes Recherches curieuses de ce temps, que je désirois qu'il achevast, n'y aiant homme en qui je m'en eusse voulu fier que de lui, lequel j'ay connu très homme de bien, fidèle et vigilant. ${ }^{46}$

Chausson partage son travail avec Fouet, dont on sait qu'il fut d'abord choisi pour être le «maistre de mes enfants ", Jérôme et Claude ${ }^{47}$, avant d'être temporairement affecté à la transcription de pièces manuscrites, dont une satire en vers de l'historien de Thou, l' Aletheia, dirigée contre le pape et la censure de l'Histoire ${ }^{48}$. Il y a aussi Culerier, un « enfant de Genève » acculé par le besoin, à qui L'Estoile confie le soin de «l'inventaire de mes livres, fait et escrit de ma main, pour me le transcrire, après avoir trayé et marqué ceux que j'ay envie de garder, et, Dieu aidant, vendre et me desfaire des autres à qui me les voudra bien paier et en aura affaire. À quoi je me suis finalement résolu pour un meilleur dessein $»^{49}$. Devant l'immensité de la tâche à accomplir et sans doute aussi pour maîtriser le coût des frais de l'opération, L'Estoile embauche un des membres de sa très nombreuse progéniture, son fils François, à qui revient la mission de recopier le manuscrit des Etrennes du roi d'Agrippa d'Aubigné que le père tenait à tout prix à conserver pour sa perfection ${ }^{50}$.

\section{Cotes}

11 Le système de cotation des livres introduit, pour sa part, le lecteur au cœur même des activités qui caractérisent la réorganisation de la réserve exceptionnellement riche de la bibliothèque stellaire au cours des années 1606 à 1611 . À ce titre, on ne compte plus le nombre de notes minutieusement consacrées aux travaux d'archivage, de tri et de classement. Cette opération avait déjà pris belle allure dès février 1608 grâce à la diligence de Chausson :

Le samedi $9^{e}$, Chausson a achevé l'inventaire des cottes des alphabets de mes pacquets, qui contiennent un ramas, presque d'un siècle, de nouvelletés et curiosités de ce temps, sur toutes sortes de matières et subjets, avec le nombre d'iceux, qui est de mille deux cents dix. Et vont jusques à la fin de l'an $1607 .{ }^{51}$ titres alphabétiquement indexés qui portent le dépôt à un total de 3392 « ramas ». Ces 
pièces sont ensuite déposées "sur les tablettes hautes de ma galerie, par $\mathrm{M}^{\mathrm{e}} \mathrm{Abraham}$ " $^{52}$, où elles rejoignent un lot constitué de 120 livres, «tant grands que petis", mais «non empacquetés $»^{53}$. À partir de ces indications, on peut déduire qu'un paquet renfermait en moyenne une trentaine de pièces. On peut dès lors reconstituer, ne serait-ce que partiellement, les différentes séries classées par L'Estoile de la façon suivante ${ }^{54}$ :

\section{Série à une lettre}

$\mathrm{Q}$ : satires et bagatelles contre le pape. Le $\mathrm{Q}$ « est la dernière lettre de mon premier alphabet » (29 août 1608, t. IX, p. 120).

U/V : pièce théologique : le Traité de l'Eglise de Duplessis-Mornay «avec les Responses et répliques, d'une part et d'autre » (31 juillet 1608, t. IX, p. 110) $)^{55}$.

$\mathrm{Z}:$ « plaisante drollerie » contre le pape (31 janvier 1609, t. IX, p. 215).

\section{Série à deux lettres}

AA: «pacquet en roulleau » de "fadèzes et superstitions du temps » et de "menues farfadelles de marchandises de Romme, avec une botte de Nostre-Dame " recueillies jusqu'à la fin de 1607 (30 août 1608, t. IX, p. 121).

DD : paquets de bulles, indulgences, pardons, confréries (7 avril 1610, t. X, p. 195).

$\mathrm{EE}$ : paquet de prières « où il y en a de toutes façons » (11 juin 1608, t. IX, p. 88).

SS : paquet de discours royaux sur l'assassinat des princes (15 décembre 1610, t. XI, p. 40). XX: «pacquets jésuistiques » (30 juin 1610, t. X, p. 298 et p. 230 et 16 mars 1611, t. XI, p. 88$)^{56}$.

YY : pièces relatives à la Ligue (29 décembre 1608, t. IX, p. 184).

\section{Série à trois lettres ${ }^{57}$}

GGG : paquet de 32 bagatelles publiées entre le $1^{\text {er }}$ juillet et le 30 septembre 1606 (15 novembre 1606 , t. VIII, p. 252).

$\mathrm{HHH}$ : paquet de 26 bagatelles ramassées entre le $1^{\mathrm{er}}$ octobre et le 31 décembre 1606 (5 janvier 1607, t. VIII, p. 268).

KKK : paquet de 31 ramas du $1^{\text {er }}$ avril au 30 juin 1607 (3 juillet 1607, t. VIII, p. 317).

$\mathrm{ZZZ}$ : « paquets de la réunion de l'Eglise » (12 avril 1610, t. X, p. 197) de 120 traités anciens et modernes (15 mai 1610, t. X, p. 240-241).

\section{Série à quatre lettres ${ }^{58}$}

AAAA : paquet de 45 lettres du $1^{\text {er }}$ janvier 1608 au 30 juin 1608 (15 juillet 1608, t. IX, p. 106).

BBBB : paquet de 43 lettres (ibid.).

CCCC : paquet de 50 « curiosités et bagatelles nouvelles » du $1^{\mathrm{er}}$ janvier au 30 septembre 1608 réunies par $\mathrm{M}^{\mathrm{e}} \mathrm{Abraham} \mathrm{en} \mathrm{une} \mathrm{seule} \mathrm{journée} \mathrm{de} \mathrm{tri} \mathrm{(10} \mathrm{octobre} 1608$, t. IX, p. 140).

DDDD : paquet de 41 bagatelles du $1^{\mathrm{er}}$ octobre au 11 novembre 1608 par $\mathrm{M}^{\mathrm{e}}$ Abraham (15 novembre 1608 , t. IX, p. 162).

EEEE : paquet de bagatelles du 11 novembre au 31 décembre 1608 par $\mathrm{M}^{\mathrm{e}}$ Abraham (5 janvier 1609, t. IX, p. 193). 
FFFF : paquet dans lequel L'Estoile insère une copie de la Lettre du duc de Saxe au roi Mathias de Hongrie qui forme la $42^{\mathrm{e}}$ de "divers recueils et escrits à la main de l'an 1608 » (31 janvier 1609, t. IX, p. 212) ${ }^{59}$.

GGGG : paquet de «mélanges bagatières » du $1^{\mathrm{er}}$ janvier au 29 février 1609 (fin février 1609 , t. IX, p. 225).

RRRR: paquet "d'écrits divers, tant imprimés que manuscrits » du $1^{\text {er }}$ janvier au 29 février 1610 (3 mars 1610, t. X, p. 161).

SSSS : paquet de "divers écrits et fadaises de ce temps » manuscrits et imprimés du $1^{\mathrm{er}}$ mars au 30 avril 1610 (30 avril 1610, t. X, p. 211).

Pour lacunaire qu'il soit ${ }^{60}$, ce tableau ne manque pas d'attirer l'attention sur la méthode de travail bibliographique et les indices de mémorisation qui doivent faciliter la consultation des « ramas $»^{61}$. À cet effet, L'Estoile dispose d'un système d'abréviations reporté sur des feuilles séparées, sur lesquelles est consigné le mouvement de ses prêts : la lettre $R$. pour signaler les livres prêtés et rendus ${ }^{62} ; S$. C. pour les pièces qui lui ont été prêtées et qu'il a rendues Sans avoir gardé de Copie ${ }^{63} ;$ le $D$. équivaut à «Donner » et le $B$. à «Bon » pour désigner un « ramas » exceptionnel ${ }^{64}$. Reportés à même le Journal, ces mêmes sigles remplissent une fonction d'aide-mémoire pour gérer efficacement la refonte du catalogue général et montrent, en tout cas, que parvenu à la fin de sa vie, l'auteur réduit progressivement la rédaction de son Journal à l'univers de ses livres. L'ultime note est sans équivoque sur ce point et montre à quel point une vision "crépusculaire " englobe l'univers de l'auteur, lorsque l'encre et le monde sont placés sur un axe réversible :» Le Mareschal d'Ancre perd cent mil pistoles. / Tout est triste, comme noirci d'encre $»^{65}$.

En tout état de cause, l'inventaire reflète l'étendue et la variété des curiosités intellectuelles, parmi lesquelles la théologie, l'histoire et les humanités imposent nettement leur supériorité ${ }^{66}$. La satire religieuse contre le pape, les superstitions, les bigoteries, les bulles, indulgences et prières occupent le premier rang en ces années 1606-1611, non loin devant la littérature politique et les thématiques du régicide, du complot des jésuites et des pamphlets de la rébellion ligueuse, soit un ensemble de textes relatifs aux menaces qui pèsent sur l'ordre public et la stabilité de l'État légitime. Sur ces préoccupations propres au Politique gallican que fut L'Estoile, viennent se greffer les visées iréniques soulevées par l'actualité religieuse et la question alors très débattue de la réunion des religions. D'innombrables "lettres", terme générique très vague, et une littérature de "bagatelles" complètent ce panorama et démontrent qu'aux yeux du collectionneur la littérature de l'éphémère a droit de cité, comme reflet de l'époque et archives pour l'avenir, au même titre que les genres nobles consacrés par l'histoire littéraire ${ }^{67}$.

\section{Du for privé à l'échange}

Cela posé, il convient d'interroger la finalité des activités déployées par L'Estoile durant toutes ces années. Pour jaloux qu'il soit de son bien, le collectionneur invétéré soustrait de moins en moins son dépôt au regard d'autrui, proches, amis, curieux et visiteurs. $\mathrm{Si}$, d'un côté, la collection amassée et la rédaction du Journal sont longtemps restées confinées dans la sphère $d u$ "for privé ${ }^{68}$, elles ménagent peu à peu autour d'elles un temps réservé à l'échange entre lettrés et circonscrivent des moments propices à la convivialité intellectuelle. Les membres de la famille figurent parmi le cercle des premiers bénéficiaires de la générosité du patriarche qui donne à une de ses filles, Louise 
${ }^{69}$, l'Exercice de l'âme fidèle de Daniel Toussain ${ }^{70}$, et à sa sœur, Marguerite, épouse Des Fourneaux, les CEuvres spirituelles du prédicateur dominicain Louis de Grenade ${ }^{71}$. Ce commerce lettré se poursuit d'ailleurs avec d'autres femmes, qui occupent dans cet échange une place particulière qu'il convient de souligner et que motive un souci incontestable de pédagogie spirituelle. Parmi elles, Mademoiselle Duret reçoit par l'intermédiaire du cousin de L'Estoile, Édouard Molé, des livres de dévotion à son domicile de Tours $^{72}$. À l'inverse, le généreux bailleur, privé des Sermons sur Job de Calvin prêtés à l'horloger du roi Gréban, emprunte finalement l'exemplaire personnel d'une de ses tantes, Mademoiselle de Fontenay, pour le lire durant les fêtes de Noël ${ }^{73}$. Échange de bons procédés, il lui donne encore son Institution de la religion chrétienne "de l'impression de Perrin, qui est la plus belle et la meilleure de toutes, et qui se recouvre rarement $»^{74}$. Et comment ne pas mentionner Camille Morel, « une de mes bonnes amies, et la perle des filles de nostre aage ", qui lui offre des vers de sa composition contre la Ligue ${ }^{75}$ ? À M. Despinelle, il confie le

gros Registre-Journal in-folio, tout escrit de ma main, contenant les choses plus mémorables avenues sous le règne de Henri III, où le bon et le mauvais, le véritable et le mesdisant, sont peslemeslés ensemble, et dont j'ay fait un livre à part du meilleur, qui est pour moi seul, et non pour autres. ${ }^{76}$.

En retour, le même Despinelle lui adresse «diverses poésies non imprimées, entre lesquelles y a Huict sonnets de Ronsard en faveur d'une des filles de la feue Roine-mère, qu'on a tirées des mains de M. Gallandius $»^{77}$. À d'autres proches, tel M. de Ménard, il prête les Actes du Concile de Trente ${ }^{78}$, tandis que M. de Lisle met à sa disposition une copie des lettres du roi du Maroc à Henri IV ${ }^{79}$, et que le contrôleur Guillon lui rend au bout de quinze mois, et à sa grande surprise, les Commentaires de Monluc: «Si je ne l'eusse trouvé sur ce registre (où je ne faus guère de les escrire), je n'eusse sceu que mon livre fust donné, car j'avois perdu la mémoire de ce que j'en avois fait. » $^{80}$

17 Élargissant le réseau des relations qui facilite l'accroissement de sa bibliothèque, mais n'arrivant pas à tempérer sa soif de nouvelles acquisitions ${ }^{81}$, L'Estoile continue à engranger les ouvrages convoités depuis longtemps. La fréquentation des bibliothèques, menée parallèlement aux visites chez les libraires, nous l'avons vu ci-dessus, comble parfois ses désirs ${ }^{82}$. Ainsi en est-il de L'Union de toutes discordes, une traduction de Bucer publiée en 1527 par Herman Bodius, « un petit vieil livre [...], il y avoit longtemps que je le cherchois, pour en estre la doctrine pure et évangélique ; et l'ay trouvé par hasard, en l'Université... > ${ }^{83}$. L'édition des Taxes des parties casuelles de la boutique du pape de 1564 lui parvient de la même manière : «Il y avoit long temps que j'en cherchois un, pour remettre en la place de celui que je bruslay à la S.-Berthelemi, craingnant qu'il me bruslast. $~_{84}$ Mais c'est aussi en fouillant dans son propre cabinet que la moisson est faste. Il exhume ainsi le manuscrit grec de son père consacré aux Acta Concilii Ephesini ; mss. e. Bibliotheca V. C. Petri Stellae, graece ${ }^{85}$. Il découvre tout aussi fortuitement le traité de l'évêque de Vérone sur les martyrs : «que j'avois en mon estude dès longtemps, et que je ne connois que par la couverture (comme beaucoup d'autres qui y sont), intitulé Sancti Zenonis Veronen,... Sermones [Vérone, 1586, 429 p.] ; je l'ay leu deux fois d'un bout à l'autre, et l'ay trouvé si pur et si beau, qu'à la seconde j'ay pris plaisir d'en extraire des passages suivans. Il est in $-4^{\circ}$, relié en parchemin, si rare pour le jourd'hui, que qui en veult avoir, faut qu'il les face venir du païs. $»^{86}$ 
Rayonnant vers l'extérieur du domicile, la « librairie » riche non seulement de ses livres, mais aussi de médailles, de tableaux, portraits, plans, cartes et d'autres curiosités, attire l'intérêt d'illustres visiteurs qui honorent de leur présence le parlementaire parisien :

Ce jour, quatre seigneurs Alemans, dont il y en avoit un prince (me dit celui qui les emmena chez moi, qui estoit un mien ami), vinrent par curiosité voir mon Estude et mon Cabinet. Ils firent cas surtout de ma Carte de Normandie, de La Guillotière (comme ils avoient bien raison, car elle est l'unique et singulière); de ma petite Mort de Pavie, qui est aussi une pièce rare ; du crayon de Poltrot [de Méré], qui tua le duc de Guise devant Orléans; des pourtraits de Luther et Melanthon, qui ne sont toutesfois grand-chose, mais qu'ils honorent fort, pour estre Protestants Luthériens. Ils m'escrivirent en leur tablettes (comme ils ont de coustume), avec ce qu'ils trouvèrent que j'avois de plus beau à leur gré. Quant à mes livres (qui estoit le meilleur), ils ne les virent que par dessus pour le peu de loisir qu'ils en eurent. ${ }^{87}$

19 Un autre jour, c'est au tour d'un Grec, Emmanuel Georgius, lecteur de l'Église de Chalcédoine, de lui soumettre un ouvrage de sa composition sur les Évangiles des dimanches et des fêtes de l'année qu'il destinait à la publication. Le visiteur souhaitait recueillir l'avis de son hôte sur l'éventuelle nécessité de reproduire les images du livre, un conseil, avoue-t-il, qu'il se gardait de demander au cardinal Du Perron et au jésuite Fronton le Duc, tous deux catholiques romains et «ennemis jurés des Graeqs et de leur doctrine ${ }^{88}$. Les deux hommes se revoient peu de temps après pour prolonger leurs discussions sur les Conciles, l'Église primitive, le Pape et l'Antéchrist. À cette occasion et comme à son habitude, L'Estoile ne manque pas de prendre des notes et de remettre à son interlocuteur une modeste somme d'argent : « et lui en eusse donné volontiers davantage, si je n'en eusse esté court aussi bien comme lui $»^{89}$. Le 15 septembre 1609 , se présentent trois curieux à son étude « et y furent trois heures, dont il m'ennuioit bien $»^{90}$. Il s'agissait $\mathrm{du}$ neveu d'un ancien ambassadeur anglais, un protestant versé en langues, de M. Bergeron, converti au catholicisme, instruit en géographie et en langues et d'un médecin, nommé Hubert, catholique, bon théologien et féru en langues orientales. Ils sont suivis, le 7 octobre, du pasteur de l'ambassadeur d'Angleterre venu lui faire ses adieux, au cours d'une séparation qui montre ce que pouvaient être la nature et la qualité des liens tissés grâce aux livres :

M. Tayler [...] me vinst voir et dire à Dieu (comme il fist) avec regret, et moy à luy : pource que, de si peu que je l'avois congneu, j'avois remarqué en cest homme une singulière modestie et humanité, accompagnée d'un vray zèle à la réunion et réformation de l'Eglise de Dieu (ce qui se trouve rarement aujourd'hui en ceux de sa profession), et croy aussi qu'en partie pour la mesme cause il m'affectionnoit beaucoup..$^{11}$

En conclusion, le classement de la «librairie » de L'Estoile aura révélé un ensemble de pratiques, de gestes et de postures qui caractérise les activités du parlementaire parisien soucieux de réorganiser sa collection de livres et de manuscrits au déclin de sa vie. Les innombrables notes que le bibliophile inscrit d'une manière quasi compulsive sur son Journal renvoient à cet égard à des préoccupations qui concernent les questions les plus ordinairement pratiques du projet, en même temps qu'elles renvoient aux idées les plus soigneusement réfléchies pour configurer avec art son précieux dépôt. Par le soin et la volonté de mener à terme son dessein, le collectionneur confère à sa bibliothèque une dimension testamentaire de première importance que traversent les enjeux socioculturels de sa démarche. Les gestes les plus quotidiens sont ainsi évoqués à côté des visites fréquentes aux libraires imprimeurs de la capitale, la fréquentation des bibliothèques et les échanges quasi ritualisés de livres et de manuscrits entretenus avec 
des amis et des érudits. La présence de secrétaires et de copistes, parfois celle de membres de la famille, marque les activités laborieuses consacrées à la transcription et au classement des documents. La restructuration du fonds est à ce prix, que complètent les visites d'hôtes illustres, parfois venus de l'étranger pour admirer le précieux trésor. Grâce à eux, la bibliothèque devient un carrefour intellectuel par lequel transite un réseau d'érudits, dont la docte sociabilité annonce la République des Lettres. Plus profondément encore, les activités de L'Estoile bibliothécaire ouvrent une large fenêtre sur la part intime de sa personnalité et la conscience qu'il a de l'utilité de ses livres, que ce soit pour la quête d'une sagesse personnelle que la lecture des Essais lui avait apprise, ou le témoignage irremplaçable que constitue ce fonds pour la postérité. C'est en ce sens qu'il faut finalement comprendre le dessein de L'Estoile, lorsqu'il réunit sa collection en un geste de préservation, au sein de laquelle les grands auteurs et les "ramas" d'actualité rangés côte à côte réalisent son projet culturel. Mais la "librairie » stellaire témoigne par là-même une autre face de son propriétaire, dont les privations, la maladie et les revers de la vie n'entament pas la foi dans les pouvoirs de l'écrit. Qu'il s'agisse d'aveux discrets consentis au détour d'une note sur la destruction prudente de livres à la Saint Barthélemy, ses relations spirituelles avec les femmes de son entourage, son pardon envers les anciens Ligueurs, ou encore sa générosité à l'égard des nécessiteux, rien n'entrave la mission qu'il s'est fixée. La transmission du savoir médiatisée par l'ensemble des livres réunis sur les étagères encombrées de sa bibliothèque est l'illustration éloquente d'un engagement mené jusqu'au bout en faveur de l'humanisme lettré.

\section{NOTES}

1. Florence Greffe et José Lothe, La vie, les livres et les lectures de Pierre de L'Estoile. Nouvelles recherches. Préface d'Henri-Jean Martin, Paris, H. Champion, 2004, p. 44-77. Le libraire Jacques Douceur habitait le quartier Saint-Séverin, Philippe Renouard, Imprimeurs et libraires parisiens du XVI $I^{e}$ siècle, Paris, Librairie A. Claudin, 1898, p. 102.

2. On estime communément à environ 4500 titres le nombre d'ouvrages amassés par L'Estoile tout au long de sa vie.

3. Advis pour dresser une bibliothèque, Paris, Rolet le Duc, 1644.

4. Sauf indications contraires, nous citons d'après l'édition de G. Brunet et al., Mémoires-Journaux, Paris, Librairie des Bibliophiles, 1875-1883, 12 vol. Le Journal du règne de Henri IV est en cours de publication, Genève, Droz, 4 volumes parus.

5. Par un jour de pluie, L'Estoile rédige en latin ses dernières volontés et confession de foi qu'il a «serré dans un des tirouers du grand bureau de mon cabinet, où est le papier de feu mon père et le mien, les révolutions de ma nativité, et autres mémoires particuliers ", 11 octobre 1608, t. IX, p. 141.

6. Voir en particulier la longue réflexion que L'Estoile consacre à ce sujet dans un passage en forme de manifeste poétique de ses Registres, Mémoires-Journaux, 2 juillet 1606, t. VIII, p. 226.

7. Nous avons abordé l'intertextualité des Essais dans «Pierre de L'Estoile et Montaigne, ou la "lecture en miettes" ", in Esculape et Dionysos. Mélanges en l'honneur de Jean Céard, Genève, Droz, 
2008, p. 157-163. Voir également Warren Boutcher, The School of Montaigne in Early Modern Europe, vol. 2, Oxford University Press, 2017, p. 72-79.

8. Mémoires-Journaux, décembre 1609, t. X, p. 103. Sur son état de santé, ses dépressions, ses souffrances physiques et la préparation à la mort, ibid., 12 avril 1607, t. VIII, p. 290 ; 8 janvier 1610 , t. X, p. 108 ; la foudre s'abat près de son étude, 19 mars 1610, t. X, p. 171 ; saignées et hébétude, $1^{\text {er }}$ mai 1610 , t. X, p. 214 ; début d'incendie dans la cuisine, 15 mai 1611, t. XI, p. 119 ; deux mois de grave maladie, 2 novembre 1610, p. t. XI, p. 24.

9. Voir Jean-Paul Pittion, Le livre à la Renaissance. Introduction à la bibliographie historique et matérielle, Turnhout, Brepols, 2013.

10. Mémoires-Journaux, 10 octobre 1608, t. IX, p. 141.

11. Montaigne se dit également fasciné par la qualité du papier chinois (Journal de voyage, éd. Fausta Garavini, Folio, 1983, p. 212) qui participe chez lui d'un imaginaire fécond de la matière, voir Elisabeth Schneikert, "Montaigne et le manuscrit chinois », dans L'Euvre et ses miniatures. L'objet autoréflexif dans la littérature européenne, dir. Luc Fraisse et Éric Wessler, Paris, Classiques Garnier [à paraître]. Nous remercions notre collègue de nous avoir spontanément communiqué son étude.

12. Il déplore la mort du libraire : «Ce jour fust mis en terre, à Paris, Abel Langelier, imprimeur, duquel la boutique, au Palais, est assez congnue et remarquée. Il est mort en la fleur de son âge », 20 janvier 1610, t. X, p. 119.

13. T. Hamilton, op. cit., p. 48-49 et 63-65.

14. Philippe Renouard, Imprimeurs et libraires parisiens $d u x V I^{e}$ siècle, Paris, t. III, Service des Travaux historiques de la Violle de Paris, 1979, p. 362.

15. 28 septembre 1607 , t. VIII, p. 343.

16. 14 août 1608, t. IX, p. 116. Voir également, 31 janvier 1609, t. IX, p. 212 et 15 janvier 1610, t. X, p. 114.

17. 2 décembre 1608, t. IX, p. 175.

18. 30 septembre 1609 , t. X, p. 30.

19. 26 février 1610 , t. X, p. 152.

20. 15 juillet 1609, t. IX, p. 288.

21. Notamment avec Charles Chastelain, Antoine $\mathrm{Du}$ Breuil, Denis Du Val, Pierre Le Bret, Guillaume Le Noir, Fédéric Morel, Claude Morillon, Robert Nivelle, Jérémie Périer, la veuve de Nicolas Roffet, Rolin Thierry...

22. 22 décembre 1608, t. IX, p. 182.

23. Voir également, 23 avril 1608, t. IX, p. 68-69 et 8 juillet 1610, t. X, p. 323 . Robinot est emprisonné peu de temps après, ibid.

24. 23 février 1610 , t. X, p. 150-151. Le père de Jean Ruel, lui aussi prénommé Jean, tenait boutique rue Saint- Jacques, à l'enseigne de la Queue de Renard. Il avait épousé la fille de l'imprimeur Pierre Haultin et sa propre fille l'imprimeur Nicolas Bonfons, voir Renouard, op. cit., p. 334-335.

25. Février 1606, t. VIII, p. 211.

26. 6 février 1610, t. X, p. 138.

27. 6 mars 1611, t. XI, p. 87.

28. Il s'agit de l'édition du P. Thomas Sanchez parue en 1607 en 2 vol. à Anvers chez M. Nutius. Voir Greffe et Lothe, op. cit., p. 950, $\mathrm{n}^{\circ} 2287$.

29. 16 janvier 1610, t. X, p. 114-115. Sur l'ouvrage de Thomas Pelletier publié par Jannon, voir Greffe et Lothe, op. cit., p. 875, $n^{\circ} 2001$.

30. Comme ce fut le cas lors du décès de «J. Guillemot, imprimeur, et libraire du Palais, le plus effronté menteur et trompeur du mestier, et le plus grand arabe et corsaire, en matière de livres, qui fust au Palais ni ailleurs ", fin février 1610, t. X, p. 153.

31. Voir la longue liste transcrite le 16 mai 1611, t. XI, p. 116-118. 
32. 12 octobre 1607, t. VIII, p. 350-351 et 8 mai 1609, t. IX, p. 261-262. Il faudrait également citer les correspondants et libraires étrangers qui sont relation avec L'Estoile, tel le marchand libraire de Leyde " que je congnois dès longtemps, nommé Elvissier ", qui lui apporte des bagatelles qu'il vient d'imprimer (27 août 1609, t. IX, p. 384).

33. 20 et 23 octobre 1608 , t. IX, p. 146-147.

34. Sur ce personnage, voir Ernest Thoinan, Les relieurs français (1500-1800). Biographie critique et anecdotique, Paris, Em. Paul, L. Huard et Guillemenin, 1893, p. 189.

35. 16 avril 1607, t. VIII, p. 289.

36. 23 mai 1607, t. VIII, p. 297 ; 4 juin 1608, t. IX, p. 84 et $1^{\text {er }}$ septembre 1608, t. IX, p. 125.

37. $1^{\mathrm{er}}$ octobre $1607, \mathrm{t}$. VIII, p. 347.

38. 15 mai 1610 , t. X, p. 238.

39. 22 février 1607, t. VIII, p. 277.

40. 23 février 1607, t. VIII, p. 277.

41. 21 juin 1607, t. VIII, p. 276.

42. 4 juin 1608, t. IX, p. 84. Rappelons que l'état dégradé du domicile de L'Estoile nécessitait de continuelles réparations.

43. Parmi les amis les plus actifs auprès de L'Estoile avec Dupuy, il y a Villiers-Hotman, Perrot d'Argenson, Peiresc et Du Breuil, dont les noms reviennent régulièrement dans le Journal.

44. 4 octobre 1607 , t. VIII, p. 348-349.

45. 15 octobre 1607 , t. VIII, p. 351.

46. 8 avril 1608, t. IX, p. 65-66. Une autre note mérite d'être citée pour les précisions qu'elles apportent sur les tâches du secrétaire : "Le jeudi $10^{\mathrm{e}}$ de ce mois [avril 1608], aiant calculé et arresté sur mon brouillas, où j'ay exactement escrit tout ce que Chosson a fait pour moy et ce qu'il m'a cousté, je trouve que, depuis le $6^{\mathrm{e}}$ aoust dernier 1607 , jusques au $7^{\mathrm{e}}$ avril de la présente année 1608, il m'a escrit vingt-neuf mains cinq feuilles de papier : à sçavoir deux gros registres, l'un de douze mains, et l'autre de huict; et deux autres commencés, d'une main et demie chacun, ou environ, transcripts, des griffonnages de mes Mémoires-Journaux de ce temps: le reste transcript des Mémoires et escrits de M. D. [u] P.[uy] et F.[lorent] C.[hrestien], et autres, en feuilles et cayers [...] Le dit Chosson n'escrit pas fort bien, mais passablement correct et sans omission, et, au reste, si viste et diligemment, qu'il me fait plus en un jour qu'un autre n'en feroit en quatre : qui a esté cause en partie de me le faire emploier, et aussi que je l'ay reconneu pour très secret, très paisible, fidèle et craingnant Dieu ", 10 avril 1608, p. 65-66.

47. 12 avril 1610, t. X, p. 198. Recommandé à L'Estoile, le jeune homme fut choisi en raison de ses "bonnes mœurs et doctrine ", ses dons d'écriture, ses aptitudes musicales et son amour de la littérature, 30 janvier 1610, t. X, p. 129. Claude de L'Estoile (1597-1652) devint membre de l'Académie française au moment de sa création.

48. 13 avril 1610, t. X, p. 197-198. Malade, Chausson mourut en décembre 1610, t. XI, p. 58.

49. 24 novembre 1608 , t. IX, p. 169. Ce travail fut achevé le 4 décembre pour quatre testons (ibid., p. 177) Voir également la transcription d'un Discours des Suisses prêté à L'Estoile par Justel, 8 janvier 1609, ibid., p. 197.

50. 27 décembre 1608, t. IX, p. 183. L'Estoile pouvait se méfier, non sans de bonnes raisons, de « l'aide » de certains de ses proches, puisque sa bibliothèque fut l'objet de larcins de la part de son fils Louis, qui «aiant crocheté mon estude, me dérobba à diverses fois tout plain de bons livres grecqs et latins, qui vendist à bon pris, m'aiant cousté bien cher », Philippe Lauer, « Le livre de raison de Louis et Pierre de L'Estoile », Bulletin de la Société de l'Histoire de Paris, 1942-1943, p. 41 (année 1589). Sur d'autres vols de livres et de médailles par ses « bons » amis, voir 19 juillet 1608 , t. IX, p. 107.

51. 9 février 1608, t. IX, p. 45.

52. 17 mai 1608, t. IX, p. 75 : passage essentiel.

53. Ibid. 
54. Le système alphabétique adopté par L'Estoile (la distinction entre $I / J, U / V$ n'est évidemment pas faite) reste relativement opaque. Il suffit de comparer les cotes des années 1608-1611 avec celles qui figurent sur l'inventaire après décès pour s'apercevoir d'une série de décalages et de disparités dans l'application alphabétique des références, voir Greffe et Lothe, op. cit., p. 70-75.

55. Sur l'inventaire après décès, la cote ne figure pas sur l'ouvrage, voir Greffe et Lothe, op. cit., p. $74, \mathrm{n}^{\circ} 756$.

56. Sur l'inventaire après décès, la cote XX est apposée sur le paquet des missels et bréviaires, Greffe et Lothe, op. cit., p. 72. On retrouve la même cote sur le paquet des "affaires de France ", ibid., p. 73.

57. Sur l'inventaire après décès apparaît uniquement la cote AAA, ibid., p. 72.

58. Aucune cote à quatre lettres ne figure sur l'inventaire après décès, ibid., p. 72.

59. Autre mention du paquet FFFF : 31 janvier 1609, t. IX, p. 215.

60. On aura remarqué que la chronologie de la cotation est intermittente (pertes, oublis, inachèvement, lacunes... ?), sans qu'il soit possible d'en déterminer la cause. On relève ainsi des paquets apparemment épars ou orphelins: les Ministromachies portant sur les superstitions religieuses (6 février 1610, t. X, p. 138) ; les Traités de controverse (19 février 1610, t. X, p. 148) ; le paquet $n^{\circ} 1^{\text {er }}$ (cérémonies officielles) qui accueille la pièce sur l'enterrement de Henri II (14 juin 1610, t. X, p. 279).

61. Voir Yann Sordet, «Une approche des "catalogues domestiques" des bibliothèques privées ( $\mathrm{XVII}^{\mathrm{e}}$-XVIII ${ }^{\mathrm{e}}$ siècles), instruments et miroirs des collections particulières ", Bulletin du Bibliophile, 1, 1997, p. 92-123. Sur les principes (pratiques) des classements médiévaux, voir Donatella NebbiaiDalla Guarda, "Classifications et classements", dans Histoire des bibliothèques françaises. Les bibliothèques médiévales du vI siècle à 1530, dir. André Vernet, Promodis-Editions du Cercle de la Libraire, 1989, p. 373-393.

62. Voir également t. VIII, p. 318, 319, 322, 326, 328.

63. T. VIII, p. 318.

64. T. XI, p. 118.

65. Septembre 1611, t. XI, p. 142.

66. Selon le calcul effectué par T. Hamilton, on obtient $36 \%$ : théologie ; $27 \%$ : histoire ; $20 \%$ : humanités ; $12 \%$ : médecine et philosophe naturelle ; $5 \%$ : droit, op. cit., p. 167.

67. Voir Gilbert Schrenck, "Prenez les bons, laissez les mauvais". Le statut du "grand" poète " dans le Journal de Pierre de L'Estoile, dans Le statut littéraire de l'écrivain, dénomination, condition, représentation, Travaux de Littérature, XX, 2007, p. 379-389.

68. Voir Au plus près du secret des cœurs? Nouvelles lectures historiques des écrits du for privé en Europe $d u X V I I^{e}$ au XVIII siècle, dir. Jean-Pierre Bardet et François-Joseph Ruggiu, Presses de l'Université Paris-Sorbonne, 2005.

69. De ses deux mariages, L'Estoile eut 18 enfants, dont trois filles se prénomment Louise, voir Greffe et Lothe, op. cit., p. 1179.

70. 4 août 1606, t. VIII, p. 235. L'Exercice de l'âme fidèle, assavoir prières et méditations pour se consoler en toutes sortes d'afflictions, s. 1., 1582, rééditée en 1583 à Francfort chez Wechel et en 1601 à Saumur chez Portau. L'ouvrage comprenait une «Préface de l'auteur aux pauvres résidus de l'Église d'Orléans ».

71. 21 septembre 1606, t. VIII, p. 243. C'est à l'occasion de son séjour à Gland, un des très rares séjours de L'Estoile en dehors de Paris. Le soin quasi maniaque qu'il prend alors pour sélectionner les ouvrages à emporter est symptomatique de son rapport passionné à la chose écrite.

72. 2 juillet 1606, t. VIII, p. 227.

73. 23 décembre 1606, t. VIII, p. 262-263.

74. 4 août 1609 , t. IX, p. 325.

75. 10 janvier 1609, t. IX, p. 199. 
76. 14 décembre 1606, t. VIII, p. 260.

77. 19 février 1607, t. VIII, p. 276. Sur la circulation manuscrite des pièces de Ronsard, voir François Rouget, Ronsard et le livre. Étude de critique génétique et d'histoire littéraire, Genève, Droz, 201, et Gregory de Rocher, «Ronsard's Dildo Sonnet : The Scandal of Poissy and Rasse des Neux », dans Writing The Renaissance. Essays on Sixteenth Century France Literature in Honor of Floyd Gray, ed. Raymond C. La Charité, French Forum, Publishers Lexington, Kentucky, 1992, p. 149-164.

78. 6 juin 1607, t. VIII, p. 302.

79. 16 août 1607 , t. VIII, p. 331.

80. 23 juin 1608, t. IX, p. 91.

81. L'Estoile est très conscient de sa dépendance ruineuse des livres et libelles. En se «dépêtrant » du lot de textes que son ami rouennais, Justel, lui propose, il reconnaît combien son " naturel y a tousjours porté et porte encores souvent, contre ma propre volonté et à regret », 10 juillet 1609, t. IX, p. 285. Et : «s'en présentant tous les jours quelque nouvelle [pièce] (dont je ne puis me descombattre, veu mon naturel, encores que $\mathrm{j}^{\prime}$ en aye bonne envie), $1^{\mathrm{er}}$ septembre 1610 , $\mathrm{t}$. X, p. 1 .

82. En compagnie de Dupuy et de Florent Chrétien, L'Estoile visite la bibliothèque du Roi installée dans le couvent des Cordeliers depuis 1604, où les trois amis admirent les livres rares, les enluminures, les manuscrits et les splendides reliures (17 octobre 1607, t. VIII, p. 352-353).

83. 22 décembre 1606, t. VIII, p. 262 et 22 août 1609: «ledit jour estant en l'Université, je rencontrai de hasard un vieil livre de devotion, que j'avois autresfois cherché, imprimé à Rouen, in- $4^{\circ}$, sans dacte ni aucteur, intitulé : L'Art de bien vivre et bien mourir, livre superstitieux, rempli de contes plus plaisans qu'édificatifs et où il y a plus à rire qu'à pleurer », t. IX, p. 346.

84. 27 juin 1607, t. VIII, p. 310. La formule finale est identique à celle que le jeune d'Aubigné prononça un jour, Sa Vie à ses enfants, éd. G. Schrenck, STFM, Nizet, 1986, p. 79.

85. 15 mai 1610 , t. X, p. 240.

86. 15 octobre 1609 , t. X, p. 63.

87. 23 février 1608, t. IX, p. 49.

88. 28 octobre 1608 , t. IX, p. 149-151.

89. 31 octobre 1608 , ibid., p. 153

90. 15 septembre 1609 , t. X, p. 16.

91. 7 octobre 1609 , t. X, p. 38-39. L'Estoile prête à l'ambassadeur de Venise son Guichardin commenté par La Noue, p. 378.

\section{RÉSUMÉS}

Le classement que L'Estoile fait de sa "librairie" à la fin de sa vie procède d'une pratique minutieuse, dont rendent compte les innombrables notes laissées par le bibliophile. Des gestes quotidiens aux visites chez les libraires, de la fréquentation des bibliothèques aux échanges de livres entre curieux, du travail des secrétaires aux visites d'hôtes lettrés, la cotation du fonds offre un tableau exceptionnellement riche d'activités et d'échanges lettrés. Elle ouvre également une large fenêtre sur les réactions intimes du collectionneur en face de sa bibliothèque et renseigne sur des réseaux de sociabilité solidement structurés autour de valeurs littéraires communes. 
Through the study of personal papers, it is understood that L'Estoile meticulously classified the books in his library. This classification sheds light on the collection's history as well as the many relationships between L'Estoile himself and his personal contacts, for example, those of book dealers, libraries, book collectors, scholars and friends. This study also reveals L'Estoile's attachment to each book and informs us of his literary social networks.

INDEX

Keywords : L'Estoile (Pierre de), library, manuscripts, actuality, classification

Mots-clés : L'Estoile (Pierre de), bibliothèque, manuscrits, actualité, classement

\section{AUTEUR}

\section{GILBERT SCHRENCK}

Université de Strasbourg, EA 1337 « Configurations littéraires »-CELAR 